\title{
Talk to us
}

The editors of Nature Ecology \& Evolution are always keen to hear about your research and answer your questions, and you can approach us at conferences or contact us via e-mail at any time.

W e are approaching the time of year with the highest frequency of scientific conferences, and, like many of our authors and readers, the Nature Ecology \& Evolution editors will be regularly on the road. Our primary goals when attending conferences are to meet researchers, to discuss the latest findings in the field and to hear about the community's publishing needs. But we can't attend every conference and are keen to reduce our environmental footprint, so we want to maximise the gains from those conferences we do attend and also explore other ways in which we can engage with the scientific community.

The most important thing to say is that editors are approachable. We are really keen to chat to everyone about their research, whether or not you have a polished manuscript. At smaller conferences, there is a good chance that we will speak to almost everyone, and we will certainly attend every talk and look at every poster, but this is harder to achieve at larger meetings. We will often chat to people whose talks we have attended or whose posters we have read, but we are equally happy to meet any attendee with an interest in the journal - please don't hesitate to contact us whether you are presenting or not. We are also very keen to listen to your feedback about Nature Ecology \& Evolution, discuss the wider publication landscape and hear your thoughts on how your discipline is and should be developing. And it is worth emphasising that we are not there just to listen to the most senior members of the community.

Our attendance at conferences is an attempt to balance disciplines and geography with the need to keep the journal sufficiently staffed. We keep a list of relevant conferences on our website, and use our Twitter account to advertise those which we will be at and to partake in the discussion under conference hashtags. Please do contact the journal (ecoevo@nature.com) or individual editors to enquire about specific conferences or to ask us to add additional conferences to the list.

We are particularly keen to demystify the editorial process, and one really useful way of doing this is through a 'meet the editors' Q\&A session. We are very happy to participate in these, and can organise them if there is interest from conference organisers or attendees. Sometimes, it might also be useful to convene a panel discussion on a topic of importance to the community, for example diversity, data availability or career options, and we are always happy to offer a journal's perspective.

In addition to conferences, we regularly visit researchers in their institutes. We often combine this with a nearby conference to be as efficient as possible, but please do let us know if you would be interested in a visit from one of our editors. We can drop in for an informal chat with just one or two researchers, or we can spend a whole day meeting an entire department. In the latter case, it's often best if we combine this with a brief lecture on demystifying the publishing process, so we can answer the sort of questions that crop up frequently. In our experience, students and postdocs find these sessions very helpful. We can also arrange to meet up elsewhere, such as at our own offices. So, please let us know if you are passing through London or Melbourne and would be interested in talking to us.

However, we do appreciate that these efforts are still likely to connect us face-to-face with only a small fraction of the community. So, we are always available via e-mail to answer queries about manuscripts or anything else you would like to discuss. We can also set up a phone or video call if that would be useful.

It's worth mentioning here what constitutes a productive pre-submission enquiry about a manuscript. Our general advice if you have a manuscript already written - whether primary research or an opinion piece - is to proceed with a formal submission through the journal's website. We are flexible about format requirements at this stage, and, by letting us see the full manuscript, you allow us to make an informed decision about whether to send it to peer reviewers. However, if you have not written the manuscript and are looking for a cautious indication of potential suitability, especially if you are unsure about the full scope of the journal, sending us a pre-submission enquiry can be useful. Ideally, we like to see a draft abstract, some key references, a key figure, if you have one prepared, and an informal cover letter explaining the nature of the advance, data and approach used in the study. There is no great art to this, however, and our ability to answer definitively will depend on the amount of information you provide, ranging from certainty about whether we will review the manuscript if we see the full draft, to a completely noncommittal suggestion that you submit a full manuscript for assessment. But each situation will be different, and we are definitely happy to discuss, via email or in direct conversation, if that is helpful to you. We are also happy to talk about any ideas you might have for posts on our Nature Research Ecology \& Evolution Community site, which is intended for more informal dissemination of ideas.

So, please do look out for us at conferences over the coming months, and reach out to us by e-mail at any time - we attach huge importance to having strong lines of communication with all parts of the community.

Published online: 29 April 2019 https://doi.org/10.1038/s41559-019-0892-4 